\title{
Subsets of a Large Cognitive Battery Better Power Clinical Trials on Early Stage Alzheimer's Disease
}

\author{
Chengjie Xiong $^{a, c}$ Hua Weng ${ }^{a}$ David A. Bennett ${ }^{d}$ Patricia A. Boyle $^{d}$ \\ Raj C. Shah ${ }^{d}$ Scot Fague $^{a}$ Charles B. Halle Richard B. Lipton ${ }^{f}$ John C. Morris ${ }^{b, c}$ \\ ${ }^{a}$ Division of Biostatistics, ${ }^{b}$ Departments of Pathology and Immunology, Department of Neurology and ${ }^{C}$ Knight \\ Alzheimer's Disease Research Center, Washington University in St. Louis, St. Louis, Mo., dRush Alzheimer's Disease \\ Center, Rush University Medical Center, Chicago, III., e Department of Epidemiology \& Population Health, and \\ fThe Saul R. Korey Department of Neurology, Albert Einstein College of Medicine, New York, N.Y., USA
}

\section{Key Words}

Alzheimer's disease $\cdot$ Clinical trial design $\cdot$ Cognitive decline $\cdot$ Mild cognitive impairment $\cdot$ Power

\begin{abstract}
Background/Aims: Cognitive batteries routinely used by the Alzheimer's disease (AD) research community may contain items that are uninformative for tracking disease progression to power clinical trials on early stage AD. We aim to identify the subsets of the most informative items from an existing cognitive battery to better power clinical trials on early AD. Methods: Longitudinal change in item scores from the battery was associated with the onset of mild cognitive impairment $(\mathrm{MCl})$ in 1,513 elderly individuals. Items whose longitudinal changes were correlated with the onset of $\mathrm{MCl}$ were selected as informative for tracking the early cognitive progression. Results: 226 items in the battery were annually assessed over a follow-up of up to 13 years. Changes of item scores over time from 187 items were significantly correlated with the onset of $\mathrm{MCl}$. For clinical trials on preclinical $A D$ and on $\mathrm{MCl}$, informative items permit smaller or similar sample sizes as compared to the entire battery, whereas uninformative items require much larger sample sizes. Conclusions:
\end{abstract}

Longitudinal changes in item scores from about $17 \%$ of items in the cognitive battery are uninformative for tracking early disease progression. Clinical trials on early $A D$ can be better powered using informative items rather than the entire battery.

(c) 2014 S. Karger AG, Basel

\section{Introduction}

Alzheimer's disease (AD) is an age-related neurodegenerative disorder that results in progressive cognitive impairment and death. Accumulating research suggests that the neurodegenerative processes associated with $\mathrm{AD}$ begin years prior to the symptomatic onset of $\mathrm{AD}$ when the disease is clinically at the early prodromal stage or a latent stage [1-3]. Many recent clinicopathologic studies have also demonstrated that asymptomatic individuals can manifest the neuropathological changes of $\mathrm{AD}$, notably senile plaques and neurofibrillary tangles [4-6]. These observations, coupled with the fact that there are currently no pharmaceutical treatments that reverse the pathological processes of $\mathrm{AD}$, have led to a major paradigm shift in the search of efficacious treatments of $\mathrm{AD}$; that is, the focus of modern $\mathrm{AD}$

\section{KARGER}

E-Mail karger@karger.com

www.karger.com/ned
(C) 2014 S. Karger AG, Basel

0251-5350/14/0432-0131\$39.50/0
Chengjie Xiong, $\mathrm{PhD}$

Division of Biostatistics, Washington University in St. Louis Campus Box 8067

St. Louis, MO 63110 (USA)

E-Mail chengjie@wubios.wustl.edu 
clinical trials now is on individuals at the earliest clinical stages, such as mild cognitive impairment [7] (MCI) and/ or very mild dementia (i.e., a clinical dementia rating [8] (CDR) of 0.5), or even the preclinical stage [9] prior to the substantial development of clinical symptoms as these may be the groups of individuals in which targeted therapies may have the greatest chance of preserving brain function.

The paradigm shift in clinical trials on $\mathrm{AD}$ subsequently has led to three major inter-related biomedical decisions that must be made by investigators in designing modern clinical trials at the early stages of $\mathrm{AD}$ : the cognitive outcome measure, sample size, and disease duration. Because cognitive batteries routinely used by the $\mathrm{AD}$ research community have been traditionally designed to track the disease progression after symptomatic onset and to identify cases of fully developed $\mathrm{AD}$ dementia in comparison to normal controls, they often show significant ceiling and floor effects. Therefore, the current cognitive batteries may contain items that are neither sensitive nor specific for tracking early stage disease progression. As a result, they only exhibit subtle changes during the very early stage or the preclinical stage of AD. Several recent randomized clinical trials (RCTs) using existing instruments (e.g., the Alzheimer's Disease Assessment Scale-Cognitive subscale [10]) failed to detect a significant decline in placebo groups with MCI. Especially for RCTs on early stage or preclinical stage of $A D$, the lack of progression on existing cognitive outcomes has become an important challenge to the feasibility of such trials because of the need for a large number of individuals to be followed over many years to allow meaningful statistical conclusions to be drawn [11-13]. Large, long-duration RCTs are time-consuming and prohibitively costly. Although emerging cerebrospinal fluid (CSF) biomarkers and neuroimaging markers [14-18] have been reported to show early changes in $\mathrm{AD}$ progression, recently revised FDA guidelines for RCTs on early stage AD mandate that treatments of AD may be approved only if they demonstrate cognitive and functional benefits (FDA Guidance for Industry Alzheimer's Disease: Developing Drugs for the Treatment of Early Stage Disease [19]: http:// www.fda.gov/downloads/Drugs/GuidanceCompliance RegulatoryInformation/Guidances/UCM338287.pdf).

The objective of this manuscript is to provide better cognitive outcomes for future RCTs on early stage $\mathrm{AD}$. We posit that subsets of items, identified from a large cognitive battery that are shown to be the most informative to early disease progression, can better power clinical trials on early stage $\mathrm{AD}$ than the other uninformative items and even the entire battery by reducing the sample sizes and improv- ing the efficiency. In order to identify the most informative items, we focused on tracking the disease progression (not necessarily for the prediction of the disease), and analyzed the longitudinal changes of item scores of individual items from the cognitive battery administered to a large longitudinal cohort study, and correlated the time to the onset of MCI with the time of the item score changes (i.e., from endorsement to non-endorsement of the item over time). Finally, we estimated the sample sizes to adequately power future RCTs on $\mathrm{MCI}$ and on preclinical $\mathrm{AD}$ using the composite cognitive scores from the items identified as the most informative for early disease progression, and compared them to the composite scores derived from the entire battery as well as the items not identified as informative.

\section{Materials and Methods}

The longitudinal cognitive database of the Rush Memory and Aging Project (MAP), a longitudinal clinical-pathologic cohort study of aging and dementia, was analyzed first for identifying the most informative items to track cognitive progression of early stage $\mathrm{AD}$ and then for powering clinical trials on early stage $\mathrm{AD}$.

\section{Participants}

Participants were individuals from the MAP (initiated in 1997), an ongoing longitudinal, community study of common chronic conditions of old age. Participants were recruited primarily from continuing-care retirement communities throughout the Chicago metropolitan area because the ability to maintain high rates of clinical follow-up and to obtain autopsy is key to the MAP mission. This was supplemented by recruitment at senior and subsidized housing, churches, and social service agencies to ensure a range of socioeconomic status, race, and ethnicity. A requirement for study entry is that participants understood what was involved in the study in order to sign an Informed Consent and agreed to donate their brains, spinal cords, nerves and muscles at the time of death [20]. A total of $n=1,513$ elderly individuals who were either cognitively normal or with MCI or AD at baseline were available for our analyses as of September 21, 2012. Out of the 1,513 individuals, 1,037 were cognitively normal, 402 had MCI and 74 had AD at baseline. The baseline characteristics of the participants are shown in table 1.

\section{Standard Protocol Approval and Patient Consents}

Written informed consent was obtained from all study participants. The study was approved by the Institutional Review Boards of Rush University Medical Center and the Washington University School of Medicine.

\section{Cognitive Assessment and Clinical Diagnosis}

Cognition was assessed annually with a comprehensive battery testing cognitive domains commonly affected by aging and AD: episodic memory, semantic memory, working memory, perceptual speed, and visual - spatial ability. Details of the cognitive function tests have been reported previously [21, 22]. In brief, 20 cognitive tests were administered annually. Seven were episodic memory measures: Word List Memory, Recall, and Recognition [23] and 
Table 1. Baseline characteristics of the sample (total, $\mathrm{n}=1,513$ )

\begin{tabular}{llll}
\hline & $\begin{array}{l}\text { Normal } \\
(\mathrm{n}=1,037)\end{array}$ & $\begin{array}{l}\text { MCI } \\
(\mathrm{n}=402)\end{array}$ & $\begin{array}{l}\text { AD } \\
(\mathrm{n}=74)\end{array}$ \\
\hline Age, y, mean \pm SD & $78.81 \pm 7.47$ & $81.91 \pm 7.44$ & $84.86 \pm 6.07$ \\
Gender, \% of female & 75.70 & 69.90 & 54.05 \\
Education, y, mean \pm SD & $14.45 \pm 3.28$ & $14.43 \pm 3.02$ & $14.09 \pm 3.92$ \\
$\begin{array}{l}\text { Race: \% for Caucasian } \\
\text { \% for African American }\end{array}$ & 93.83 & 90.80 & 94.59 \\
\% of others & 5.40 & 7.96 & 5.41 \\
$\begin{array}{l}\text { APOE4 positive, \% } \\
\text { MMSE, mean } \pm \text { SD }\end{array}$ & 0.68 & 0.75 & 0 \\
$\begin{array}{l}\text { Global cognition, } \\
\quad\end{array}$ & $28.41 \pm 1.70$ & 27.61 & 32.43 \\
\multicolumn{1}{c}{ mean \pm SD } & $0.26 \pm 0.46$ & $-0.41 \pm 0.49$ & $-1.51 \pm 0.74$ \\
\hline
\end{tabular}

immediate and delayed recall of Story A from Logical Memory of the Wechsler Memory Scale - Revised [24] and of the East Boston Story $[25,26]$. Semantic memory was assessed with a 15 -item version [21] of the Boston Naming Test [27], Verbal Fluency [23, 26], and a 15-item version [26] of the National Adult Reading Test [28]. Working memory tests included Digit Span Forward and Digit Span Backward [24] and digit ordering [26, 29]. Four measures of perceptual speed were administered: the oral version of the Symbol Digit Modalities Test [30], Number Comparison [26, 31], and two measures from a modified version [22] of the Stroop Neuropsychological Screening Test [32]: number of color names correctly read in 30 s minus the number of errors and number of colors correctly named in 30s minus the number of errors. Visuospatial ability was assessed with a 15-item version of Judgment of Line Orientation [33] and a 16-item version of Standard Progressive Matrices [34]. In addition, the Mini-Mental State Examination (MMSE [35]) was also used to serve as a brief measure of cognitive function. To minimize floor and ceiling artifacts and other sources of measurement error, a global composite measure of cognition that was previously reported $[21,22]$ and based on all 20 tests including MMSE and measures of episodic memory, semantic memory, working memory, perceptual speed, and visuospatial ability was created by converting raw scores to $\mathrm{z}$ scores, using the baseline mean and SD across the entire cohort, and averaging the $\mathrm{z}$ scores. Further information about the individual tests and the derivation of the composite measure was described elsewhere $[21,22]$. Cognitive testing was scored by a computer and reviewed by a neuropsychologist to determine cognitive impairment. Participants were then evaluated by a clinician for a medical history and a neurologic examination to diagnose AD using National Institute of Neurological and Communicative Disorders and Stroke - Alzheimer's Disease and Related Disorders Association criteria [36]. The diagnosis of dementia required a history of cognitive decline and impairment in at least 2 cognitive domains, and MCI (all types) required the presence of cognitive impairment in the absence of dementia [20].

\section{Types of Item Scores}

Individual items were scored as binary for most cognitive tests except for five items (Color Name, Logical Memory IA and IIA, Verbal Fluency, and Words Correctly Read), which could not be reasonably decomposed into meaningful binary items and there- fore were treated as single-item tests with the original count score. Table 2 presents the number of individual items as well as baseline summary statistics for each of the 20 cognitive tests (including MMSE). A few items from several tests were not originally scored as binary (e.g., one item in MMSE has an original score of 0-5), such items were treated as binary in the item analyses using the perfect score vs. others. Sensitivity analyses were also done with other possible cutoffs for these items.

\section{Other Covariates}

Demographics such as age, sex, and years of education were recorded at the study entry. APOE genotyping was done and individuals were dichotomized into those with at least 1 or more copies of the E4 allele (E4 positive) vs. those without an E4 copy (E4 negative).

\section{Statistical Analysis}

A two-step procedure was implemented to select the most informative items with binary score (i.e., endorsement and non-endorsement of the item, oriented in the way that non-endorsement always indicates problems with cognition) from the entire cognitive battery for better tracking the disease progression and designing future RCTs at the early stages. The first step examined the longitudinal changes of scores for each individual item and tested whether the item score changes over time were associated with the onset of MCI. Specifically, for each individual, we first computed the age at onset of MCI. If an individual never developed MCI or $\mathrm{AD}$ during the entire follow-up, the age at onset was considered right-censored. If a subject was already classified as MCI or AD at the baseline, the age at onset of MCI was considered interval-censored between 0 and the age at baseline. For each individual and each binary item, we then computed the age of non-endorsement defined as the age when the item was incorrectly endorsed. Because of the fluctuation of item level scores over time, the item-specific age of non-endorsement for each individual also was considered interval censored with the left side of the interval as the first age in the follow-up when the item was endorsed incorrectly and the right side as the age at the first occurrence of non-endorsement after which the item remained incorrectly endorsed over time [37]. If an individual already incorrectly endorsed the item at baseline, the left side of the age of non-endorsement was defined as 0 . If an individual never incorrectly endorsed the item during the entire follow-up, the age of non-endorsement was considered right-censored at the age of last assessment. For each item, the item-specific age of non-endorsement and the age at onset of MCI were then correlated across the entire cohort including subjects who were normal, MCI, or AD at baseline. The correlation was estimated by a Kendall's coefficient of concordance through a bivariate smooth estimate of the joint density on the logarithms of the two time scales that was obtained using a mixture of Gaussian densities fixed on a grid with weights determined by a penalized likelihood approach $[37,38]$. Items with a significant correlation $(\mathrm{p}<0.05)$ were identified as informative for tracking early disease progression.

Five tests in the cognitive battery could not be reasonably decomposed as binary items because their scores are counts that can be any non-negative integers: Color Name, Logical Memory IA and IIA, Verbal Fluency (with 2 items, Animal and Fruit), and Words Correctly Read. Preliminary analyses similar to those used for the binary items were conducted using different cutoffs, suggesting that all five tests have reasonable sensitivity and specificity for tracking early disease changes. Thus, they (a total of 6 items) 
Table 2. Item characteristics of the cognitive tests at baseline and the number of informative items identified

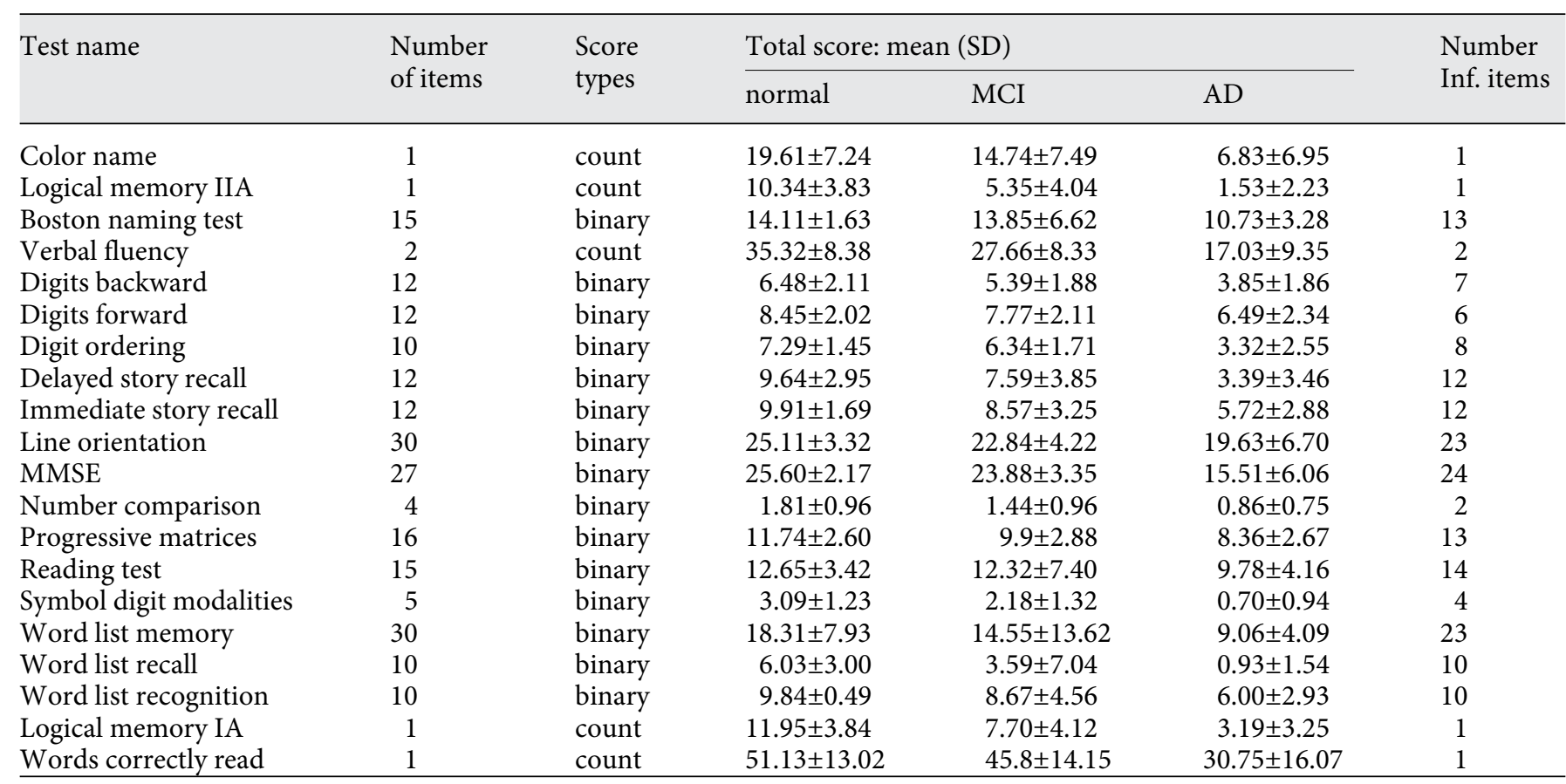

$\mathrm{SD}=$ Standard deviation; Inf. = informative.

were always included as informative items, using their original scores (not the dichotomized scores).

Because for each item, the correlation between the age of nonendorsement and the age at onset of MCI does not indicate whether the age of non-endorsement occurred earlier or later than the age at onset of MCI, the second step of our item selection procedure compared the center of the interval censored age of non-endorsement and age at onset of MCI, and identified the items whose age of non-endorsement occurred later than the age at onset MCI for at least more than half (e.g., $>51 \%$ ) of individuals in the entire cohort. This subset of the informative items was used for tracking the disease progression and designing RCTs on individuals who were already MCI at baseline.

\section{Power Analysis}

In traditional prevention trials, it is common to randomize high-risk subjects to active drug or placebo and analyze the time taken for the onset of the disease as the primary efficacy endpoint with a standard Cox proportional hazards model (PHM) [39]. Recently, for modern RCTs either on 'preclinical AD' or on MCI, it has been suggested that the PHM approach is subject to a loss of power to detect a treatment effect, in comparison to the approach with a linear mixed model for repeated measures (MMRM [40]) on a well-defined cognitive composite [41]. Based on the informative items identified, three cognitive composite scores were computed similar to the way the global cognitive composite score was defined (see Cognitive Assessment and Clinical Diagnosis): one using all informative items, another using the subset of the informative items whose age of non-endorsement occurred later than the age at onset of MCI, and the other using the items that were not identified as informative (i.e., uninformative). For each composite, a mixed model for repeated measures (MMRM [40]) was then implemented to estimate the mean 2-year, 3-year, and 4-year change from the baseline as well as the relevant variance and covariance parameters from subjects who were considered 'preclinical AD' (in the absence of biomarker data, operationally defined as those who were cognitively normal at baseline but with at least 1 copy of APOE E4 allele) or MCI at baseline. To examine how the newly formed cognitive composites with informative items influence the sample sizes required for adequately powering future RCTs at the early stages, these estimates were used to further estimate the sample sizes for future RCTs on 'preclinical AD' or MCI with either 2-year, 3-year, or 4-year annual follow-ups using a standard normal test [42]. A power of $80 \%$ was assumed for all power analyses. For comparison purpose, similar power analyses were also conducted using the same subjects sample but the global cognitive composite derived from the entire cognitive battery as well as the cognitive composite derived from items not selected as informative. All statistical analyses were implemented in SAS [43].

\section{Results}

A total of 226 items (including 220 items with binary data and 6 items from 5 tests with count data) from the cognitive battery were analyzed. Of the 1,037 cognitively 
Table 3. Estimated mean change (standard error, SE) of cognitive composites from baseline on individuals with MCI and 'Preclinical AD'

\begin{tabular}{|c|c|c|c|c|}
\hline $\begin{array}{l}\text { Types of items used } \\
\text { in the composite }\end{array}$ & Clinical stage & $\begin{array}{l}\text { Years of } \\
\text { follow-up } \\
\text { (sample size) }\end{array}$ & $\begin{array}{l}\text { Mean change } \\
\text { (SE) from } \\
\text { baseline }\end{array}$ & $\begin{array}{l}\text { Ratio } \\
\text { of mean/SE }\end{array}$ \\
\hline Informative items & $\begin{array}{l}\text { Preclinical AD } \\
\text { MCI }\end{array}$ & $\begin{array}{l}2 y(n=139) \\
3 y(n=121) \\
4 y(n=102) \\
2 y(n=275) \\
3 y(n=212) \\
4 y(n=174)\end{array}$ & $\begin{array}{l}-0.085(0.030) \\
-0.093(0.031) \\
-0.197(0.036) \\
-0.261(0.038) \\
-0.416(0.048) \\
-0.558(0.060)\end{array}$ & $\begin{array}{l}-2.833 \\
-3.000 \\
-5.472 \\
-6.868 \\
-8.667 \\
-9.300\end{array}$ \\
\hline Uninformative items & $\begin{array}{l}\text { Preclinical AD } \\
\text { MCI }\end{array}$ & $\begin{array}{l}2 y(n=139) \\
3 y(n=121) \\
4 y(n=102) \\
2 y(n=275) \\
3 y(n=212) \\
4 y(n=174)\end{array}$ & $\begin{array}{l}-0.034(0.030) \\
-0.034(0.035) \\
-0.083(0.040) \\
-0.072(0.028) \\
-0.144(0.040) \\
-0.206(0.045)\end{array}$ & $\begin{array}{l}-1.133 \\
-0.971 \\
-2.075 \\
-2.571 \\
-3.600 \\
-4.578\end{array}$ \\
\hline All tests & $\begin{array}{l}\text { Preclinical AD } \\
\text { MCI }\end{array}$ & $\begin{array}{l}2 y(n=139) \\
3 y(n=121) \\
4 y(n=102) \\
2 y(n=275) \\
3 y(n=212) \\
4 y(n=174)\end{array}$ & $\begin{array}{l}-0.063(0.024) \\
-0.082(0.028) \\
-0.182(0.033) \\
-0.129(0.022) \\
-0.244(0.030) \\
-0.330(0.036)\end{array}$ & $\begin{array}{l}-2.625 \\
-2.929 \\
-5.515 \\
-5.863 \\
-8.133 \\
-9.167\end{array}$ \\
\hline
\end{tabular}

normal individuals, 404 developed MCI (all types) or AD in the period of up to 12 years of follow-up. Individual items with very limited longitudinal data (i.e., those with less than $30 \%$ of the total annual assessments across the entire cohort for a total of 34 items) were excluded from the item analyses. The first step of the two-step procedure identified a total of 181 binary items whose age of nonendorsement is significantly correlated with the age at onset of MCI, resulting in a total of 187 informative items including the 6 items from 5 tests with count data. At the second step, a subset of 62 items were further identified for tracking disease progression on individuals who had MCI at baseline because their age of non-endorsement occurred later than the age at onset of MCI for at least more than half (e.g., >51\%) of individuals in the entire cohort. The last column of table 2 presents the number of informative items identified from each of the 20 cognitive tests in the battery. Online supplementary table 2A (for all online suppl. material, see www.karger.com/doi/ $10.1159 / 000365733$ ) lists all individual items that were found to be informative for tracking the early disease progression.

Using a consistent way of forming cognitive composites (i.e., averaging the $\mathrm{z}$ scores across multiple tests ob- tained by using the baseline mean and SD of each test), four cognitive composites were computed using the following: the 187 informative items as identified above, the 62 informative items whose age of non-endorsement occurred later than the age at onset of MCI, all 20 cognitive tests in the battery, and the items not identified as informative by our analyses. Table 3 presents the estimated mean change and associated standard error (SE) of the cognitive composites from baseline among subjects with 'preclinical AD' at baseline, as well as among those with MCI at baseline. Results in table 3 suggest that, for both 'preclinical AD' and MCI, the rate of change on the cognitive composite from the informative items is larger than that on the cognitive composite based on the entire battery, which in turn is larger than that on the cognitive composite based on items not identified as informative.

To assess the ability of the items identified as informative for tracking early disease progression to improve the design of modern RCTs on early stage $\mathrm{AD}$, we considered two types of future two-arm RCT to test the cognitive efficacy of a novel therapeutic compound against a placebo on individuals who were 'preclinical AD' or MCI at baseline. The sample size ratio of the RCTs is assumed 1:1 between the two arms. The longitudinal follow-ups are as- 
Table 4. Sample sizes of future RCTs on 'Preclinical AD' and MCI

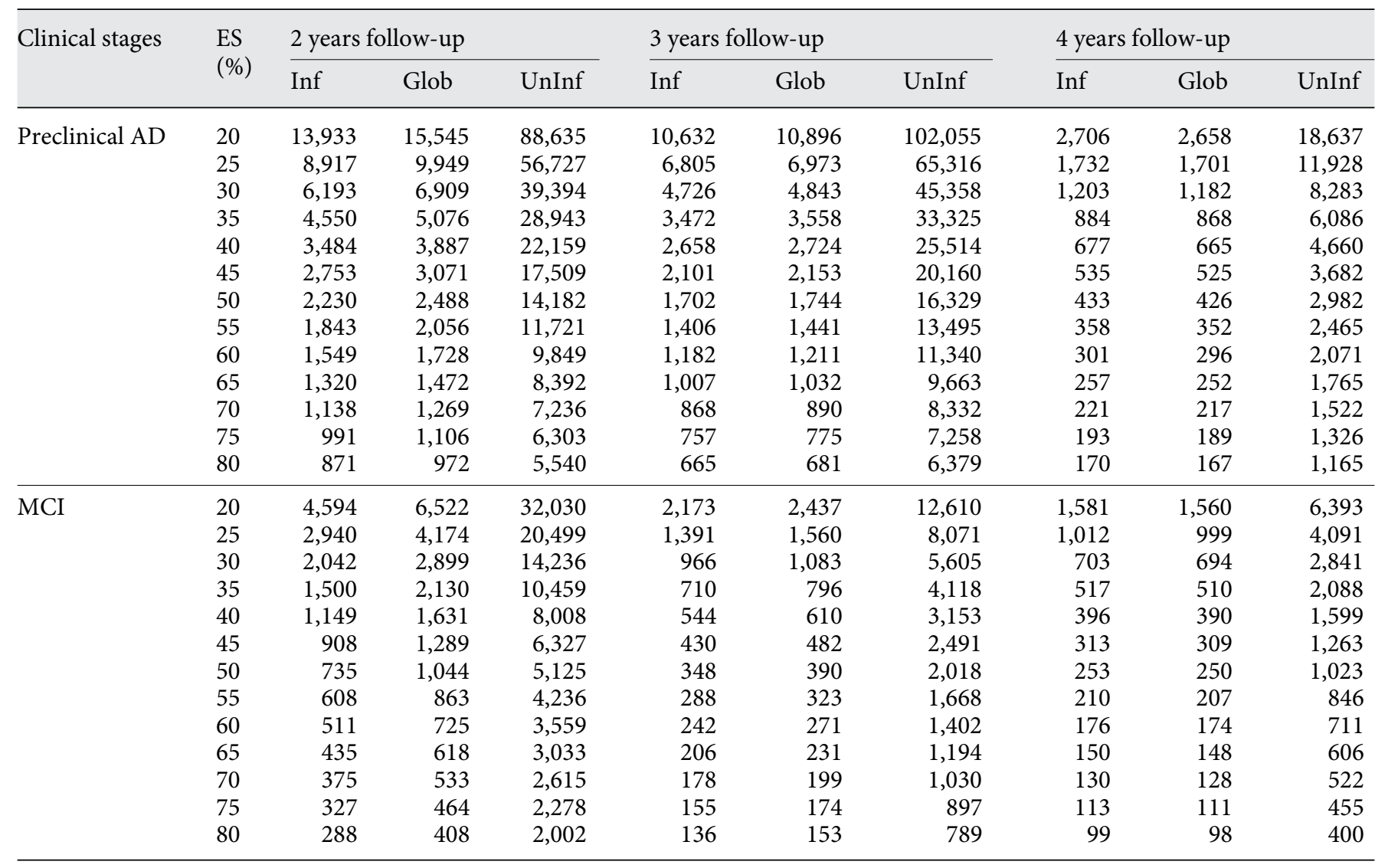

Inf = Cognitive composite using informative items alone; UnInf = cognitive composite using uninformative items; Glob = cognitive composite using all 20 tests.

sumed to be annual with a range of 2 to 4 years. The effect size (ES) of the novel treatment is assumed as a percentage of improvement on the change from baseline as compared to the placebo, the latter of which was estimated by an MMRM assuming a covariance structure of compound symmetry. We used change on the cognitive composite from the 187 informative items as the primary efficacy endpoint for the RCT on 'preclinical AD', and change on the cognitive composite from the subset of 62 informative items as the primary efficacy endpoint for the RCT on MCI because these items' age of non-endorsement occurred later than the age at onset of MCI. As indicated in table 4, for the trial on 'preclinical AD' with a 2 -years or 3-years annual follow-up, the use of 187 informative items provides smaller sample sizes than the use of entire cognitive battery, resulting in a reduction of sample size from 2 to $10 \%$ across a wide range of effect sizes. On the other hand, the sample size of the trial using items not identified as informative is at least 6 times of that using the informative items. For example, with a reasonable effect size of $40 \%$, the RCT of 2-year follow-up can be adequately powered with a total of 3,484 subjects using the informative items and 3,887 subjects using the entire battery, and 22,159 subjects using the items not identified as informative. For the RCT on 'preclinical AD' with a 4-years follow-up using informative items, the sample size is slightly lower than that of using the entire battery, but is only about one seventh of that using items not identified as informative. Very similar observations can be made for the RCTs on MCI. In comparison to the entire battery, the 62 informative items provide a sample size reduction of $11-30 \%$ in a RCT on MCI with a 2 or 3 -years of follow-up, and only a slight increase (about 1\%) in a RCT on MCI with a 4-years follow-up. On the other hand, the items not identified as informative require sample sizes that are 4-7 times that of the informative items. 


\section{Discussion}

Individual items are the foundation for cognitive outcome measures used in RCTs on $\mathrm{AD}$, and their test scores are inherently noisy when used to longitudinally track cognitive progression, especially at the early stages of disease. To our best knowledge, our item level longitudinal analyses represent the first comprehensive effort to associate the onset of early cognitive symptoms (i.e., MCI) with longitudinal change in item level scores from a comprehensive cognitive battery. We found that, out of a total of 226 items from a large cognitive battery administered longitudinally in MAP on a large sample size of 1,513 individuals, the longitudinal item score changes were associated with the onset of MCI for 187 items over an annual follow-up of up to 13 years. Of these, the item score changes for 62 items (i.e., from endorsement to non-endorsement of items) occurred after the onset of MCI. A total of 39 items (i.e., 17.26\%) were found to be uninformative for tracking the cognitive progression at early disease stage, that is, the longitudinal changes of item scores were not associated with the onset of MCI.

Although the conventional test scores from cognitive batteries used in many $\mathrm{AD}$ studies have been very successful in cross-sectional discrimination of fully developed symptomatic AD from normal aging, they are less satisfactory in longitudinally tracking the early changes of $\mathrm{AD}$ when individuals are at the early or preclinical stage of the disease. In fact, when some of current cognitive tests are administered to individuals in the preclinical or early stage of the disease, the resulting data are subject to enormous ceiling and floor effects. Because cognitive items and tests with significant ceiling and floor effects have limited use in tracking longitudinal changes, they are unlikely to be correlated with the early disease progression, and therefore have limited power to predict whether or at what time point an individual will develop subtle sign of early changes, which will eventually lead to the onset of MCI and AD.

There is currently a major conundrum in the search of effective treatments of AD. On the one hand, accumulating research evidence indicates that neurodegenerative processes associated with $\mathrm{AD}$ begin years prior to the symptomatic onset of $\mathrm{AD}$ [4-6], suggesting that the optimum time window for treatment interventions is when the disease is clinically at the early prodromal stage or even the latent or preclinical stage. On the other hand, the lack of detection of progression by cognitive tests routinely used in current $\mathrm{AD}$ research makes the sample size for clinical trials on early disease stage or preclinical AD a formidable task to achieve. This bottleneck is primarily due to the lack of cognitive measures that can reliably detect the earliest possible cognitive changes of early-stage $\mathrm{AD}$ in the presence of high inherent inter-individual variability during the progression of the disease [44]. This question is challenging because, by definition, longitudinal cognitive changes have to be subtle during the early stages of the disease.

In comparison to the entire battery, we found that the 187 informative items (in the form of a standard composite) provide smaller or comparable sample sizes to adequately power future RCTs on individuals with 'preclinical $\mathrm{AD}$ ', operationally defined as, in the absence of biomarker data in this analysis, those who were cognitively normal at baseline but with at least one allele of APOE4. For future RCTs on MCI, a subset of 62 informative items whose item score changes occurred later than the onset of MCI also provides sample sizes smaller than or comparable to those from the entire battery. Importantly, we found several folds of increased sample sizes to power future RCTs on either 'preclinical AD' or MCI when items not identified as informative by our analyses were used. These results have 3 major implications in designing future RCTs on early stage $\mathrm{AD}$ and in tracking early disease progression. First, they suggest that the commonly used cognitive batteries with years of longitudinal data remain the most important pilot data to design future RCTs on early-stage $\mathrm{AD}$, as a majority of the items were informative to early disease progression, consistent with several reports (albeit cross-sectional) [45-49]. Second, given the current challenges facing modern RCTs on early-stage $\mathrm{AD}$ in terms of choosing appropriate cognitive outcomes and determining the adequate sample sizes, our results suggest the feasibility of using subsets of informative items from an existing cognitive battery as the cognitive efficacy outcome in powering future RCTs on 'preclinical AD' or MCI. Third, when it comes to tracking early disease progression, data collected on a large number of uninformative items may represent less than optimal use of precious research resources as well as an increased burden to research participants. In fact, as demonstrated by our results, the uninformative items in the battery dramatically reduce the power of the informative items in tracking early progression and designing RCTs at the early stage, partly due to the decreased rate of change and the inflated variance because of the contamination from the items not informative to early disease changes.

It is important to point out that the items that were not identified as informative (i.e., uninformative items) by our analyses do not automatically become invalid items from the cognitive battery. All these items have face valid- 
ity and may be useful in tracking the disease progression at other stages. Even at the early disease stage, their composite score also shows some cognitive decline on subjects with MCI or 'preclinical AD' as presented in table 3, albeit at a much lesser degree when compared to the informative items. Our results should therefore not be interpreted as against the use of these items in AD research. For example, the parent MAP study that provided the data performs annual clinical evaluations on participants until death; thus, items that are sensitive to change among persons with moderate-to-severe dementia are also needed.

Our study has many strengths. The participants were community-dwelling and examined with annual home visits. Thus, many of the biases inherent in getting persons to be evaluated in a clinic setting are reduced. The overall participation rate exceeded $90 \%$ over the entire duration of the follow-up period, reducing bias that results from attrition. The large pool of items from 20 cognitive performance tests is among the largest item pools currently available in community-based prospective cohort studies. This allowed us to identify a large number of items of potential utility in RCTs on preclinical AD and MCI.

Our study also has limitations. The study cohort is selected; the generalizability of the findings needs to be established through independent studies, especially those with a population-based longitudinal design. Further, although it is the most cost effective to utilize an existing longitudinal cognitive database to select most informative items to track early disease progression, neuropsychological theory-based development of outcome measures on prospectively designed longitudinal studies is needed to fully establish the validity and psychometric properties of cognitive outcomes that can serve as the primary efficacy endpoint of future RCTs on early stage AD. Next, our analyses were based on the cognitive data already collected according to a well-established protocol [20]. Therefore, the effect of order, presentation, and possibly interference $[50,51]$ of the cognitive testing on our findings cannot be adequately addressed. In addition, our analytic approach was based on the technique of survival analysis, which implicitly assumed that everyone will develop $\mathrm{MCI} / \mathrm{AD}$ if he or she lives long enough. Although this assumption may not be entirely unreasonable, its impact on the analysis results warrants further investigation. Finally, biomarker data would be needed to more accurately define 'preclinical AD', and our results need to be further validated when biomarker data are available.

\section{Acknowledgement}

The authors thank RUSH MAP Clinical Core for subject assessments. This study was supported by National Institute on Aging (NIA) grant R01 AG034119 for Chengjie Xiong. This study was also partly supported by the NIA grant P50 AG05681, P01 AG03991, P01AG26276, and U01 AG032438 for Chengjie Xiong and John Morris, RO1 AG17917 and AG34374 for David Bennett, Patricia Boyle, and Raj Shah. The data set used in this manuscript can be requested to RUSH MAP and is available from the corresponding author after appropriate approval.

\section{Appendix}

Informative items for designing RCTs on early $\mathrm{AD}$ (187 on 'Preclinical AD', and 62 on MCI) and their description.

\section{References}

1 Bateman RJ, Xiong C, Benzinger T, et al: Clinical and biomarker changes in dominantly inherited Alzheimer's disease. N Engl J Med 2012;367:795-804.

2 Sperling RA, Aisen PS, Beckett LA, et al: Toward defining the preclinical stages of $\mathrm{Al}$ zheimer's disease: recommendations from the National Institute on Aging - Alzheimer's Association workgroups on diagnostic guidelines for Alzheimer's disease. Alzheimers Dement 2011;7:280-292.

3 Katzman R: Editorial: the prevalence and malignancy of Alzheimer's disease. A major killer. Arch Neurol 1976;33:217-218.

4 Price JL, McKeel DW, Buckles VD, Roe CM, Xiong C, Grundman M, Hansen LA, Petersen RC, Parisi JE, Dickson DW, Smith CD, Davis DG, Schmitt FA, Markesbery WR, Kaye J,
Kurlan R, Hulette C, Kurland BF, Higdon R, Kukull W, Morris JC: Neuropathology of nondemented aging: presumptive evidence for preclinical Alzheimer's disease. Neurobiol Aging 2009;30:1026-1036.

5 Bennett DA, Schneider JA, Arvanitakis Z, Kelly JF, Aggarwal NT, Shah RC, Wilson RS: Neuropathology of older persons without cognitive impairment from two communitybased studies. Neurology 2006;66:1837-1844.

-6 Morris JC, Price JL: Pathologic correlates of nondemented aging, mild cognitive impairment, and early stage Alzheimer's disease. J Mol Neurosci 2001;17:101-118.

7 Petersen RC, Doody R, Kurz A, Mohs RC, Morris JC, Rabins PV, Ritchie K, Rossor M, Thal L, Winblad B: Current concepts in mild cognitive impairment. Arch Neurol 2001;58:1985-1992.
8 Morris JC: The Clinical Dementia Rating (CDR): current version and scoring rules. Neurology 1993;43:2412-2414.

9 Morris JC, Roe CM, Xiong C, Fagan AM, Goate AM, Holtzman DM, Mintun MA: APOE predicts A $\beta$ but not tau Alzheimer's pathology in cognitively normal aging. Ann Neurol 2010;67:122-131.

-10 Mohs RC, Knopman D, Petersen RC, Ferris SH, Ernesto C, Grundman M, Sano M, Bieliauskas L, Geldmacher D, Clark C, Thal LJ: Development of cognitive instruments for use in clinical trials of antidementia drugs: additions to the Alzheimer's Disease Assessment Scale that broaden its scope. The Alzheimer's Disease Cooperative Study. Alzheimer Dis Assoc Disord 1997;11(suppl 2):S13-S21. 
$>11$ Ringman JM, Grill J, Rodriguez-Agudelo Y, Chavez M, Xiong C: Prevention trials in persons at-risk for dominantly-inherited $\mathrm{Al}$ zheimer's disease: opportunities and challenges. Alzheimers Dement 2009;5:166-171.

-12 DeKosky ST, Fitzpatrick A, Ives DG, Saxton J, Williamson J, Lopez OL, Burke G, Fried L, Kuller LH, Robbins J, Tracy R, Woolard N, Dunn L, Kronmal R, Nahin R, Furberg C; GEMS Investigators: The Ginkgo Evaluation of Memory (GEM) study: design and baseline data of a randomized trial of Ginkgo biloba extract in prevention of dementia. Contemp Clin Trials 2006;27:238-253.

13 Launer LJ: Prevention of AD: the which, when, and on whom? Alzheimer Dis Assoc Disord 2006;20(3 suppl 2):S75-S78.

14 Hampel H, Frank R, Broich K, Teipel SJ, Katz RG, Hardy J, Herholz K, Bokde ALW, Jessen F, Hoessler YC, Sanhai WR, Zetterberg H, Woodcock J, Blennow K: Biomarkers for Alzheimer's disease: academic, industry and regulatory perspectives. Nat Rev Drug Discov 2010;9:560-574.

-15 Fagan A, Roe C, Xiong C, Mintun M, Morris JC, Holtzman D: Cerebrospinal fluid tau/A42 ratio as a prediction of cognitive decline in nondemented older adults. Arch Neurol 2007;64:343-349.

16 Shaw LM, Vanderstichele H, Knapik-Czajka M, Clark CM, Aisen PS, Petersen RC, Blennow K, Soares H, Simon A, Lewczuk P, Dean R, Siemers E, Potter W, Lee VM, Trojanowski JQ; Alzheimer's Disease Neuroimaging initiative: Cerebrospinal fluid biomarker signature in Alzheimer's disease neuroimaging Initiative subjects. Ann Neurol 2009;65:403413.

17 Mintun MA, LaRossa GN, Sheline YI, Dence CS, Lee SY, Mach RH, Klunk WE, Mathis CA, DeKosky ST, Morris JC: $\left[{ }^{11} \mathrm{C}\right]$ PIB in a nondemented population: potential antecedent marker of Alzheimer's disease. Neurology 2006;67:446-452.

18 Chen K, Reiman EM, Alexander GE, Bandy D, Renaut R, Crum WR, Fox NC, Rossor MN: An automated algorithm for the computation of brain volume change from sequential MRIs using an iterative principal component analysis and its evaluation for the assessment of whole-brain atrophy rates in patients with probable Alzheimer's disease. NeuroImage 2004:22:134-143.

19 http://www.fda.gov/downloads/Drugs/Guid anceComplianceRegulatoryInformation/ Guidances/UCM338287.pdf.

-20 Bennett DA, Schneider JA, Buchman AS, Barnes LL, Boyle PA, Wilson RS: Overview and findings from the rush Memory and Aging Project. Curr Alzheimer Res 2012;9:646663.

21 Wilson RS, Barnes LL, Bennett DA: Assessment of lifetime participation in cognitively stimulating activities. J Clin Exp Neuropsychol 2003;25:634-642.
22 Wilson RS, Barnes LL, Krueger KR, Hoganson G, Bienias JL, Bennett DA: Early and late life cognitive activity and cognitive systems in old age. J Int Neuropsychol Soc 2005;11:400407.

23 Morris JC, Heyman A, Mohs RC, et al: The Consortium to Establish a Registry for $\mathrm{Al}$ zheimer's Disease (CERAD). Part I. Clinical and neuropsychological assessment of $\mathrm{Al}$ zheimer's disease. Neurology 1989;39:11591165 .

24 Wechsler D: Wechsler Memory Scale - Revised manual. New York: Psychological Corporation, 1987.

25 Albert MS, Smith LA, Scherr PA, et al: Use of brief cognitive tests to identify individuals in the community with clinically diagnosed $\mathrm{Al}$ zheimer's disease. Int J Neurosci 1991;57: 167-178.

26 Wilson RS, Beckett LA, Barnes LL, Schneider JA, Bach J, Evans DA, Bennett DA: Individual differences in rates of change in cognitive abilities of older persons. Psychol Aging 2002; 17:179-193.

27 Kaplan EF, Goodglass H, Weintraub S: The Boston Naming Test. Lea \& Febiger; Philadelphia, 1983.

28 Nelson HE: National Adult Reading Test (NART): test manual. Windsor, UK: NFER Nelson, 1982

29 Cooper JA, Sagar HJ: Incidental and intentional recall in Parkinson's disease: an account based on diminished attentional resources. J Clin Exp Neuropsychol 1993;15: 713-731.

30 Smith A: Symbol Digit Modalities Test manual - revised. Los Angeles: Western Psychological, 1982.

31 Ekstrom RB, French JW, Harman HH, Dermen D: Manual for kit of factor-referenced cognitive tests. Princeton, NJ: Educational Testing Service, 1976.

32 Trenerry MR, Crosson B, DeBoe J, Leber WR The Stroop Neuropsychological Screening Test. Psychological Assessment Resources, Odessa, FL, 1989

-33 Benton AL, Varney N, et al: Visuospatial judgment: a clinical test. Arch Neurol 1978; 35:364-367.

34 Raven JC, Court JH, Raven J: Standard progressive matrices, 1992 edition; Raven manual: Section 3. Oxford: Oxford Psychologists Press, 1992.

35 Folstein MF, Folstein SE, McHugh PR: 'MiniMental State': a practical method for grading the mental state of patients for the clinician. J Psychiatr Res 1975;12:189-198.

36 McKhann G, Drachman D, Folstein M, Katzman R, Price D, Stadlan EM: Clinical diagnosis of Alzheimer's disease: report of the NINCDS-ADRDA Work Group under the auspices of Department of Health and $\mathrm{Hu}$ man Services Task Force on Alzheimer's disease. Neurology 1984;34:939-944.
37 Bogaerts K, Lesaffre E: Estimating local and global measures of association for bivariate interval censored data with a smooth estimate of the density. Stat Med 2008;27:59415955.

38 Bogaerts K, Lesaffre E: Modeling the association of bivariate interval-censored data using the copula approach. Stat Med 2008;27:63796392.

39 Lawless JF: Statistical models and methods for lifetime data. Wiley, New York, 1982.

40 Diggle PJ, Heagerty P, Liang K-Y, Zeger SL: Analysis of Longitudinal Data, ed 2. New York: Oxford University Press, 2002.

41 Donohue MC, Gamst AC, Thomas RG, Xu R, Beckett L, Petersen RC, Weiner MW, Aisen P; Alzheimer's Disease Neuroimaging Initiative: The relative efficiency of time-to-threshold and rate of change in longitudinal data. Contemp Clin Trials 2011;32:685-693.

-42 Xiong C, Zhu K, Yu K, Miller JP: Statistical Modeling in Biomedical Research: Longitudinal Data Analysis; in Rao CR, Philip Miller J, Rao DC (eds): Handbook of Statistics: Epidemiology and Medical Statistics. Amsterdam: Elsevier BV, 2008.

43 SAS Institute, Inc. SAS/STAT ${ }^{\circledR}$ 13.1, User's Guide, Cary, NC, 2013.

44 Stern Y, Jacobs DM: Preliminary findings from the predictors study: utility of clinical signs for predicting disease course. Alzheimer Dis Assoc Disord 1995;9:S14-S18.

$\checkmark 45$ Grober E, Buschke H, Crystal H, Bang S, Dresner R: Screening for dementia by memory testing. Neurology 1988;38:900-903.

46 Backman L, Jones S, Berger AK, et al: Cognitive impairment in preclinical Alzheimer's disease: a meta-analysis. Neuropsychology 2005; 19:520-531

47 Hall CB, Derby C, LeValley A, Katz MJ, Verghese J, Lipton RB: Education delays accelerated decline on a memory test in persons who develop dementia. Neurology 2007;69:16571664.

48 Hall CB, Lipton RB, Sliwinski M, Katz MJ, Derby CA, Verghese J: Cognitive activities delay onset of memory decline in persons who develop dementia. Neurology 2009;73:356361.

49 Twamley EW, Legendre Ropacki SA, Bondi MW: Neuropsychological and neuroimaging changes in preclinical Alzheimer's disease. J Int Neuropsychol Soc 2006;12:707-735.

50 Rowe G, Hasher L, Turcotte J: Interference, aging, and visuospatial working memory: the role of similarity. Neuropsychology 2010;24: 804-807.

51 Beglinger LJ, Gaydos B, Tangphao-Daniels O, Duff K, Kareken DA, Crawford J, Fastenau PS, Siemers ER: Practice effects and the use of alternate forms in serial neuropsychological testing. Arch Clin Neuropsychol 2005;20: 517-529. 